\title{
A Parallel Elastic Haptic Thimble for Wide Bandwidth Cutaneous Feedback
}

\author{
Daniele Leonardis ${ }^{(凶)}$, Massimiliano Gabardi, Massimiliano Solazzi, \\ and Antonio Frisoli \\ Percro Laboratory, Scuola Superiore Sant'Anna of Pisa, Pisa, Italy \\ d.leonardis@santannapisa.it \\ https://www.santannapisa.it/it
}

\begin{abstract}
Design of wearable fingertip haptic devices is often a compromise between conflicting features: lightness and compactness, against rich and neat haptic feedback. On one side direct drive actuators (i.e. voice coils) provide a clean haptic feedback with high dynamics, with limited maximum output forces. On the other side mechanical transmissions with reduction can increase output force of micro sized motors, at the cost of slower and often noisy output signals. In this work we present a compact fingertip haptic device based on a parallel elastic mechanism: it merges the output of two differently designed actuators in a single, wide bandwidth haptic feedback. Each actuator is designed with a different role: one for rendering fast, high frequency force components, the other for rendering constant to low frequency components. In the work we present design and implementation of the device, followed by experimental characterization of its performance in terms of frequency response and rendering capabilities.
\end{abstract}

Keywords: Cutaneous feedback $\cdot$ Haptics $\cdot$ Bandwidth · Fingertip • Wearable $\cdot$ Parallel elastic

\section{Introduction}

In recent years, rendering of the sense ot touch in teleoperated or virtual reality has become a rich field of research, especially concerning highly wearable haptic devices. In particular the scientific literature shows the development of different devices able to provide the user with a specific cutaneous feedback, such as thermal [5], vibratory [14], contact orientation [2], contact force [7], or a combination of the mentioned feedback $[4,16]$. In [12], a complete review of portable and wearable haptic devices for the fingertips can be found.

Rendering the correct physical interaction is a challenging objective [1], and concerning portable and wearable haptic devices, practical requirements such as wearability and portability of the devices determine limits to features of the rendered feedback. Limitations can be, for instance, in terms of bandwidth and 


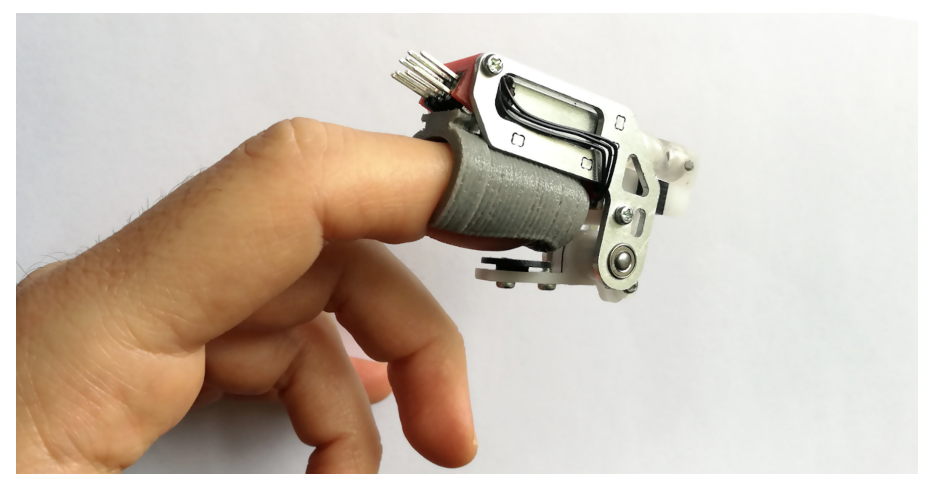

Fig. 1. The presented Parallel Elastic Thimble, featuring two actuators coupled by an elastic element to render from static to high frequency cutaneous feedback

force amplitude for force rendering devices, range of motion for shape rendering thimbles or heat flux for thermal devices. The choice of the actuation system is a trade-off between feedback performance (max. force, bandwidth, max. stroke, noise) and device requirements (mass, dimensions, wearability). In fact, the actuation system is usually the heaviest part of a portable fingertip haptic interface. Typical electromagnetic actuators (DC motors or voice coils) used in haptic devices allow for high quality haptic rendering, ranging from constant to high frequency force components, with the drawback of a limited maximum output force. Small actuators provided with mechanical reduction can be used to amplify the output force, yet at the cost of reduced output bandwidth and degraded quality of the haptic feedback in terms of noise and backlash. A different solution to increase the feedback bandwidth while keeping a reasonable constant force is obtained by coupling in a serial manner a macro-actuator (or a small reduced actuator) featuring low bandwidth and high output force, with a micro-actuator, with wide bandwidth but low output force. Such a solution has been explored in grounded haptic devices in a series configuration, i.e. a very compact "micro" actuator is placed at the end-effector of a desktop or grounded haptic device $[9,11,15]$ to enhance its dynamic response. Concept of parallel micro-macro configuration via elastic parallel transmission was proposed in [10] and then developed for robotic arm manipulators in [13] and [17]. To the knowledge of authors, micro-macro solutions have never been applied to a fully wearable and compact fingertip haptic display. In this paper, the proposed novel fingertip device features a parallel elastic actuation system, obtained by combining the output of two micro DC motors of the same size: one with high mechanical reduction and considerable continuous force, the second with low reduction and high dynamics of the output force (Fig. 1). 


\section{The Parallel Elastic Thimble}

The fingertip device proposed in this work is conceived to render 1 dof cutaneous feedback at the fingerpad, featuring rendering of the no-contact to contact transition and modulation of the contact force. In order to efficiently render from static to high-frequency force components, a parallel elastic configuration implementing two parallel micro-sized motors has been experimented. Overview of the design and placement of the different components is shown in Fig. 2. Actuators have been placed on the finger dorsum, and the whole device design has been studied in order to minimize lateral interference with other fingers and interference with the hand workspace. The device is $16 \mathrm{~mm}$ wide and weights $21 \mathrm{~g}$. Importantly, the slim design at the sides of the device allow for easy switching between thimbles of different sizes. Different thimble sizes were fabricated by 3D printing in TPU (Thermoplastic Polyurethane) soft polymer, resulting in a compliant and precise fit of the device to the specific finger shape, not requiring additional fastening elements (velcro or clips). A slider mechanism allows easy and rapid switch between different thimbles (Fig. 3).

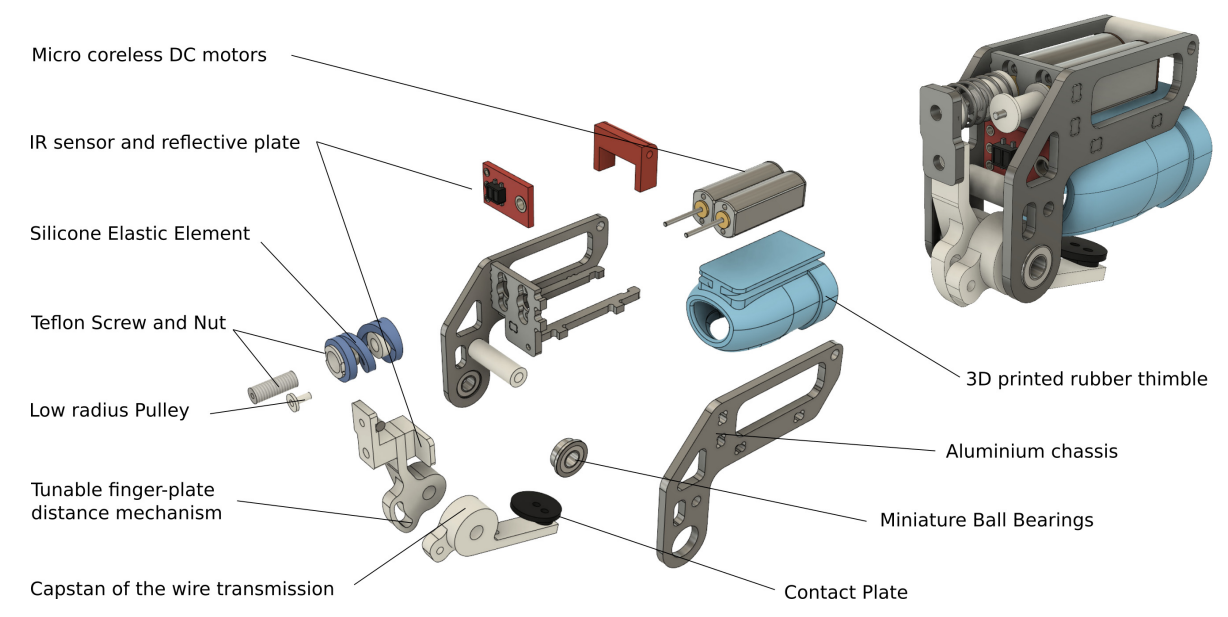

Fig. 2. Mechanical components of the Parallel Elastic Haptic Thimble

The moving plate in contact with the fingerpad has been implemented through a 1 dof link with a revolute joint. A slot mechanism with a fastening screw has been designed in order to tune distance of the plate with respect of the finger surface, thus minimizing the required displacement of the plate. Considering that a displacement of few millimeters of the contact plate is a sufficient range in cutaneous haptic devices $[6,8]$, the consequent angular displacement, due to the revolute joint, can be negligible ( $18 \mathrm{~mm}$ radius). Also, the revolute joint with miniaturized ball bearings allow to minimize friction with respect to a linear slider mechanism. 

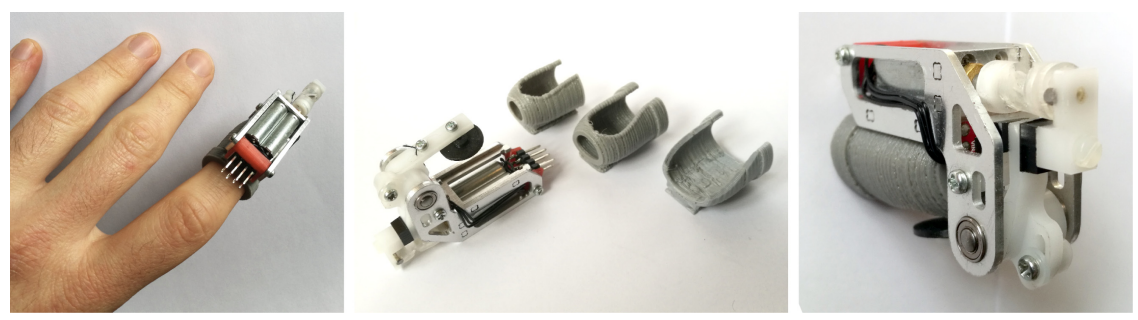

Fig. 3. The slim lateral profile of the device (left) allows easy switch of rubber thimbles with different finger sizes through a slider mechanism (right)

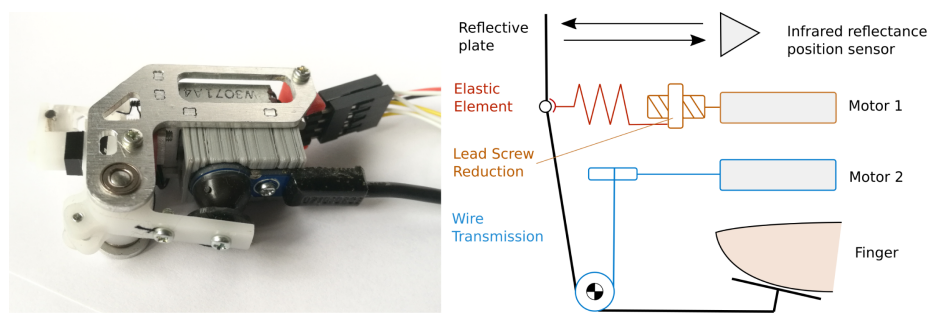

Fig. 4. Scheme of the transmission mechanism (left) and detail of the force sensor mounted in place of the fingertip for characterization of the device

\subsection{Actuation Scheme}

The parallel elastic actuation scheme is shown in Fig. 4 (left). As a first prototype of the parallel elastic concept design, we decided to implement two identical actuators of the same size, varying only the mechanical reduction between each of them and the moving plate. Actuators were two Minebea K30 micro DC motors, diameter $8 \mathrm{~mm}, 5 \mathrm{~V}$ nominal voltage. The output of the first actuator is connected through a lead-screw mechanism and an elastic element to the moving link. The lead-screw obtains a high mechanical reduction, although with no-backdrivability. Importantly, the use of the lead-screw has been chosen in order to avoid introduction of sources of noise, as it would happen, in example, for a more conventional gear reduction. The second actuator is coupled to the moving link by means of a one branch wire transmission: a capstan (radius $8 \mathrm{~mm}$ at the moving link is connected by the actuation wire to a pulley (radius $1 \mathrm{~mm}$ ) at the output shaft of the motor. It results in a low reduction, highly reversible mechanical transmission. The elastic element has been fabricated from a silicon tube: after preliminary experiments it was preferred with respect to a steel spring due to the inherent presence of a damping factor. A position sensor has been embedded into the device, measuring displacement of the moving link with the contact plate. We used a reflectance infrared sensor, due to its very compact size, to the measurement range particularly suitable for the device and to the sensitivity of the sensor to small displacement (measured noise of $0.01 \mathrm{~mm}$ in the middle point of the measuring range). 


\section{Experimental Characterization}

The experimental activity was conducted to characterize the novel (for the size of a fingertip mechanism) compact parallel elastic structure in terms of frequency response and output forces and displacement. A holder for a compact force sensor (Optoforce $10 \mathrm{~N}$ with resolution of $1 \mathrm{mN}$ ) was fabricated to be mounted in place of the rubber thimble (Fig. 4 left). A microcontroller board (Teensy 3.6) was used to implement the low level control of the device, to acquire the analog infrared position sensor, and to drive motors through a dual H-bridge (Texas Instruments DRV8835). Sample time of the low-level control loop was $1 \mathrm{KHz}$. Communication with a host PC was implemented through a Wiz5500 Ethernet module and UDP communication. A Matlab Simulink Desktop-Real Time model, executed on the host PC, implemented the high level control interface and data recording.

The first experimental activity consisted in measuring the force output of the two actuators. The contact plate was positioned at the contact threshold with the force sensor. Then, a slow voltage reference ramp was commanded to each motor separately for ten repetitions. The obtained current intensity to force characteristics are shown in Fig. 5 (left). The graph highlights the different mechanical reduction of the two identical actuators: the first obtains a higher output force, presenting non-linearity due to friction of the lead-screw mechanism. The second actuator shows a lower output force and a more linear characteristic. A position control loop was then tuned for the first actuator, in order to control displacement of the moving plate. Step response of the position control loop is shown in Fig. 5 (middle). Bandwidth of the two actuators was then measured. The first actuator was controlled in closed loop with a chirp reference position signal, ranging from 0.5 to $40 \mathrm{~Hz}$. The obtained frequency response shows a limit of the first actuator bandwidth at $15 \mathrm{~Hz}$ (Fig. 5, right). The second actuator was commanded in open loop with a chirp voltage reference ranging from 5 to $250 \mathrm{~Hz}$. The second actuator response shows a cutoff frequency of $120 \mathrm{~Hz}$, which is about
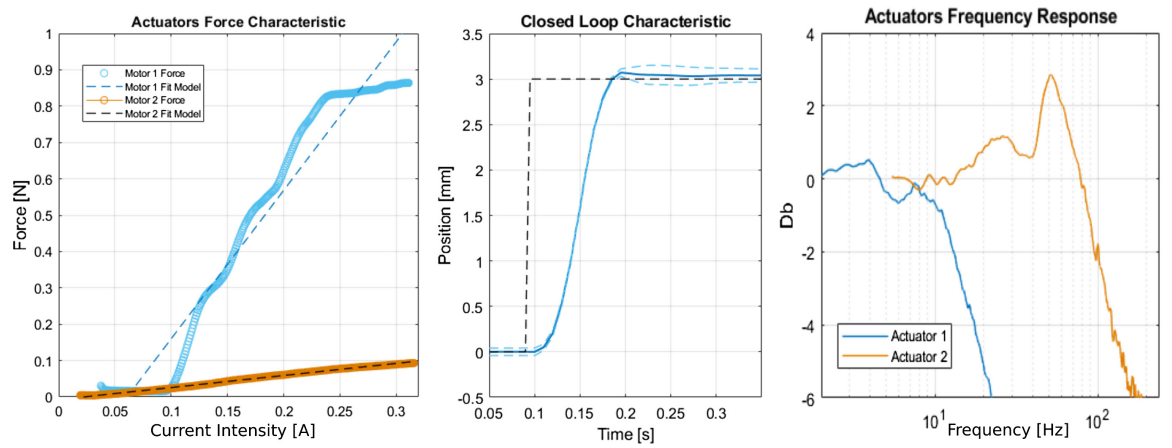

Fig. 5. Force characterization of the two actuators (left), closed loop step response of the first actuator (middle) and frequency response of the two actuators (right) 
one order of magnitude greater than the first actuator. Also, a peak appears at $55 \mathrm{~Hz}$, possibly due to a resonant frequency introduced by the elastic element of the system.

\subsection{Experimental Evaluation of Sample Texture Rendering}

Overall device performance was finally evaluated using a pre-recorded signal involving contact with a texturized surface. The device was evaluated by wearing it onto the experimenter's index finger. Displacement measurements of the contact plate were recorded through the embedded infrared position sensor. The texture pattern was taken from the "Sandpaper 100" object of the texture library of the Penn Haptic Texture Toolkit [3].
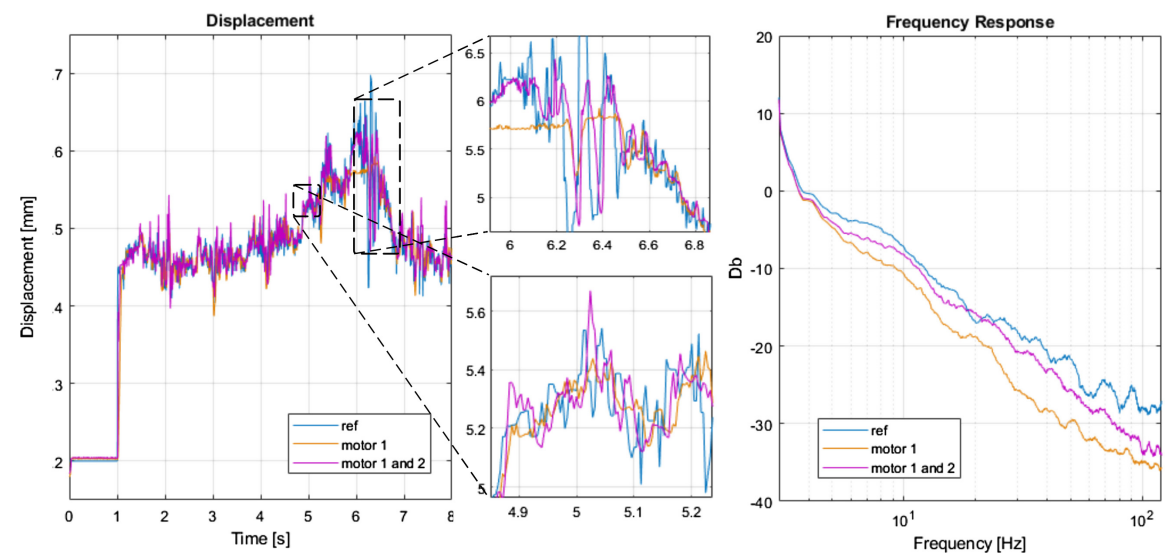

Fig. 6. Bench test of the device rendering a sample texture (sandpaper) with contact transition and non-zero constant component of the normal force

For the position-controlled actuator, normal force was converted to displacement, approximating stiffness of the fingerpad to a constant value of $0.5 \mathrm{~N} / \mathrm{mm}$. In order to gain full advantage of the parallel configuration, force reference for the second actuator was high-pass filtered at the cutoff frequency of the first actuator. With this method, the second actuator was not in charge of rendering the constant to low frequency components of the normal force, which in the simulated signal had a noticeably high value. Two data acquisition were performed: with both actuators enabled, and with the first actuator only enabled. Results are shown in Fig. 6. The benefit of both the actuators can be noticed from the frequency response (Fig. 6 (right)), closer to the reference, and from details of Fig. 6 (middle). The output of second actuator produces more crisp and reactive dynamics of the plate, whereas the first actuator alone, especially at the higher indentation levels (top detail), tends to a more flat response. 


\section{Conclusions}

The novel design of a wearable fingertip device implementing a parallel elastic mechanism was proposed. The idea was originated from the contrasting requirements of fingertip haptic devices, involving compactness and wearability of the device and rendering of relatively high constant forces together with wide bandwidth tactile cues. The parallel structure allows to optimize each actuator for different purposes: the first, with high mechanical reduction, for rendering static to low frequency cues, which in typical applications can be noticeably high (i.e. when grasping a virtual object or exploring a surface). The second actuator, with low reduction and high transparency, was designed to render high frequency tactile cues, which typically have a reduced amplitude with respect to the static and slow force components.

The obtained prototype included two miniaturized motors with noiseless mechanical reduction (a lead-screw and a capstan wire transmission) and an elastic element to couple the two actuators. Mechanical design of the prototype was focused on enhancing wearability by minimizing mass and dimensions (16 mm total width, $21 \mathrm{~g}$ mass), by optimizing arrangement of actuators, and by implementing a user's tailored and switchable soft thimble design.

Force characterization and frequency response confirmed the desired different behavior of the two actuators (same motors with different reduction) in complementary frequency ranges. A resonant peak was noticeable, and further investigation is required in order to obtain a more flat frequency response. A deeper study of the mechanical model of the system can guide the choice of the elastic element stiffness, with the aim of optimizing interaction between the two actuators.

The final evaluation with the sample texture evidenced how the reference signal can be conveniently split between the two actuators. Although more investigation is required to obtain proper optimization of the developed device, the proposed method can result in more compact wearable devices with better energy efficiency and better capabilities, in terms of quality of the output signal and hi-fidelity rendering.

\section{References}

1. Caldwell, D.G., Tsagarakis, N., Wardle, A.: Mechano thermo and proprioceptor feedback for integrated haptic feedback. In: 1997 Proceedings of the IEEE International Conference on Robotics and Automation, vol. 3, pp. 2491-2496. IEEE (1997)

2. Chinello, F., Malvezzi, M., Pacchierotti, C., Prattichizzo, D.: Design and development of a 3RRS wearable fingertip cutaneous device. In: 2015 IEEE International Conference on Advanced Intelligent Mechatronics (AIM), pp. 293-298. IEEE (2015)

3. Culbertson, H., Lopez Delgado, J.J., Kuchenbecker, K.J.: The Penn haptic texture toolkit for modeling, rendering, and evaluating haptic virtual textures (2014) 
4. Gabardi, M., Leonardis, D., Solazzi, M., Frisoli, A.: Development of a miniaturized thermal module designed for integration in a wearable haptic device. In: 2018 IEEE Haptics Symposium (HAPTICS), pp. 100-105. IEEE (2018)

5. Gallo, S., Rognini, G., Santos-Carreras, L., Vouga, T., Blanke, O., Bleuler, H.: Encoded and crossmodal thermal stimulation through a fingertip-sized haptic display. Front. Robot. AI 2, 25 (2015)

6. Gleeson, B.T., Horschel, S.K., Provancher, W.R.: Design of a fingertip-mounted tactile display with tangential skin displacement feedback. IEEE Trans. Haptics 3(4), 297-301 (2010)

7. Leonardis, D., Solazzi, M., Bortone, I., Frisoli, A.: A wearable fingertip haptic device with 3 DoF asymmetric 3-RSR kinematics. In: 2015 IEEE World Haptics Conference (WHC), pp. 388-393. IEEE (2015)

8. Leonardis, D., Solazzi, M., Bortone, I., Frisoli, A.: A 3-RSR haptic wearable device for rendering fingertip contact forces. IEEE Trans. Haptics 10(3), 305-316 (2016)

9. Lu, T., Pacoret, C., Hériban, D., Mohand-Ousaid, A., Regnier, S., Hayward, V.: Kilohertz bandwidth, dual-stage haptic device lets you touch brownian motion. IEEE Trans. Haptics 10(3), 382-390 (2016)

10. Morrell, J.B., Salisbury, J.K.: Parallel-coupled micro-macro actuators. Int. J. Robot. Res. 17(7), 773-791 (1998)

11. Pacchierotti, C., Prattichizzo, D., Kuchenbecker, K.J.: Cutaneous feedback of fingertip deformation and vibration for palpation in robotic surgery. IEEE Trans. Biomed. Eng. 63(2), 278-287 (2015)

12. Pacchierotti, C., Sinclair, S., Solazzi, M., Frisoli, A., Hayward, V., Prattichizzo, D.: Wearable haptic systems for the fingertip and the hand: taxonomy, review, and perspectives. IEEE Trans. Haptics 10(4), 580-600 (2017)

13. Shin, D., Sardellitti, I., Khatib, O.: A hybrid actuation approach for human-friendly robot design. In: 2008 IEEE International Conference on Robotics and Automation, pp. 1747-1752. IEEE (2008)

14. Solazzi, M., Frisoli, A., Bergamasco, M.: Design of a novel finger haptic interface for contact and orientation display. In: 2010 IEEE Haptics Symposium, pp. 129-132. IEEE (2010)

15. Wall, S.A., Harwin, W.: A high bandwidth interface for haptic human computer interaction. Mechatronics 11(4), 371-387 (2001)

16. Wang, D., Ohnishi, K., Xu, W.: Multimodal haptic display for virtual reality: a survey. IEEE Trans. Ind. Electron. 67(1), 610-623 (2019)

17. Zinn, M., Khatib, O., Roth, B., Salisbury, J.K.: Large workspace haptic devicesa new actuation approach. In: 2008 Symposium on Haptic Interfaces for Virtual Environment and Teleoperator Systems, pp. 185-192. IEEE (2008) 
Open Access This chapter is licensed under the terms of the Creative Commons Attribution 4.0 International License (http://creativecommons.org/licenses/by/4.0/), which permits use, sharing, adaptation, distribution and reproduction in any medium or format, as long as you give appropriate credit to the original author(s) and the source, provide a link to the Creative Commons license and indicate if changes were made.

The images or other third party material in this chapter are included in the chapter's Creative Commons license, unless indicated otherwise in a credit line to the material. If material is not included in the chapter's Creative Commons license and your intended use is not permitted by statutory regulation or exceeds the permitted use, you will need to obtain permission directly from the copyright holder. 